\title{
Master equations for two qubits coupled via a nonlinear mode
}

\author{
Rosanna Migliore • Matteo Scala • \\ Anna Napoli · Antonino Messina
}

Received: 26 April 2012 / Accepted: 15 December 2012

(C) Springer Science+Business Media Dordrecht 2013

\begin{abstract}
A microscopic master equation describing the dynamics of two qubits coupled via a nonlinear mediator is constructed supposing that the two qubits, as well as the nonlinear mode, interact, each with its own independent bosonic bath. Generally speaking the master equation derived in this way represents a more appropriate tool for studying the dynamics of open quantum systems. Indeed we show that it is more complex than the phenomenological master equation, constructed simply adding ad hoc dissipative terms.
\end{abstract}

Keywords Open quantum systems $\cdot$ Master equations $\cdot$ Dissipation $\cdot$ Decoherence $\cdot$ Entanglement

\section{Introduction}

In recent decades, due to the rapid development of experimental techniques, many schemes aimed at the controlled generation and manipulation of multipartite quantum states have been experimentally realized and theoretically analyzed [1-9]. In this context, Josephson junction-based architectures are among the best candidates due to their scalability and to the possibility of controlling in situ their dynamics by tuning external control parameters. Generally speaking, these Josephson junction-based two-level systems (qubits) can be directly coupled exploiting their mutual inductance or capacitance or via an auxiliary device playing the role of an entanglement mediator. The coherent coupling between two superconducting qubits has been realized, for example, exploiting their interaction with a monochromatic radiation field (described as a linear resonator) or by a superconducting quantum interference device (SQUID)-based mediator, which also makes it possible to measure the final state of the system in situ. In this case, the SQUID-based mediator is characterized by a nonlinearity that, as indicated in a recent paper [10], results in a strong enhancement of the entanglement established between the two qubits almost up to its maximal value. Within this context it is necessary to investigate the robustness of the theoretical entanglement generation scheme against

R. Migliore

Institute of Biophysics, National Research Council, via Ugo La Malfa 153, 90146 Palermo, Italy

M. Scala $(\varangle) \cdot$ A. Napoli · A. Messina

Dipartimento di Fisica, Universitá di Palermo, via Archirafi 36, 90123 Palermo, Italy

e-mail: matteo.scala@unipa.it

A. Messina

e-mail: antonino.messina@unipa.it 
losses that, generally speaking, corrupt the unitary scheme. To this end, the starting point is the construction of a microscopic master equation. It has indeed been demonstrated [11,12] that simply adopting phenomenological master equations can be inappropriate for describing the dynamics of systems especially when structured reservoirs are considered.

In this paper we consider a system of two qubits coupled via a nonlinear resonator supposing that each of them interacts with a bosonic reservoir. Our aim is to construct the microscopic master equation by which the entanglement dynamics of the two-qubit system can be appropriately investigated. The paper is structured as follows. The physical system and its Hamiltonian are described in Sect. 2. The microscopic master equation is presented in Sect. 3, whereas the dynamics of the system of the two qubits is investigated in Sect. 4. A few concluding remarks are given in Sect. 5.

\section{The physical system and its Hamiltonian}

Denoting by $H_{Q 1}$ the Hamiltonian of the first qubit, $H_{Q 2}$ the Hamiltonian of the second qubit, $H_{R}$ the Hamiltonian of the nonilinear resonator, and $H_{Q-R}$ the qubit-resonator interaction Hamiltonian, the Hamiltonian $H_{S}$ describing the tripartite physical system under scrutiny (assuming $\hbar=1$ ) is as follows:

$$
\begin{aligned}
H_{S} & =H_{Q 1}+H_{Q 2}+H_{R}+H_{Q-R} \\
& =\sum_{i=1}^{2} \frac{\omega_{i}}{2} \sigma_{z}^{(i)}+\omega\left(a^{\dagger} a+\frac{1}{2}\right)+\alpha\left(a^{2}+a^{\dagger 2}\right)+\sum_{i=1}^{2} \beta_{i}\left(a+a^{\dagger}\right)\left(\sigma_{+}^{(i)}+\sigma_{-}^{(i)}\right),
\end{aligned}
$$

where $\omega_{i}$ is the free frequency of the $i$ th qubit $(i=1,2), \omega$ the frequency of the bosonic mode, $\sigma_{z}^{(i)}$ the standard Pauli operator, and $a$ and $a^{\dagger}$ the annihilation and creation bosonic operators, respectively. The parameter $\alpha$ in Eq. (1) measures the strength of the nonlinearity of the bosonic mode, while $\beta_{i}$ is the coupling constant between the $i$ th qubit and the mediator.

It is well known that, in order to derive a microscopic master equation, the first step is to diagonalize the Hamiltonian of the system, explicitly finding its eigenvalues and eigenvectors [13, p. 133]. To this end we look for transformations that linearize the Hamiltonian (1). Let us consider operator $A$ defined as

$A=\frac{1}{2}\left(a S+a^{\dagger} D\right)$,

with

$S=\sqrt{\frac{\tilde{\Omega}}{\Omega}}+\sqrt{\frac{\Omega}{\tilde{\Omega}}}, \quad D=\sqrt{\frac{\tilde{\Omega}}{\Omega}}-\sqrt{\frac{\Omega}{\tilde{\Omega}}}$,

and $\tilde{\Omega}^{2}=\Omega^{2}+4 \alpha \Omega$, where $\Omega=(\omega-2 \alpha)$ (with $\left.\alpha<\omega / 2\right)$. It is immediate to verify that, by definition, $\left[A, A^{\dagger}\right]=1$ [14, p. 489]. Exploiting the new annihilation and creation operators $A$ and $A^{\dagger}$, the Hamiltonian $H_{R}$ describing the nonlinear mediator can be cast in the form

$\tilde{H}_{R}=\tilde{\Omega}\left(A^{\dagger} A+\frac{1}{2}\right)$.

To make our approach physically consistent, we confine our analysis to the case $-\omega / 2<\alpha<\omega / 2$, thereby ensuring that the condition $\tilde{\Omega}^{2}>0$ is satisfied.

Moreover, expressing the Hamiltonian describing the interaction between each qubit and the nonlinear bus in terms of the new operators $A$ and $A^{\dagger}$, we obtain 
$\tilde{H}_{Q-R}=\sum_{i=1}^{2} K_{i}\left(A+A^{\dagger}\right)\left(\sigma_{+}^{(i)}+\sigma_{-}^{(i)}\right)$,

with $K_{i}=\beta_{i} \sqrt{\tilde{\Omega} / \Omega}$. Let us suppose that $K_{i} \ll \tilde{\Omega}$. Under this condition it is legitimate to perform the well-known rotating wave approximation (RWA) obtaining

$\tilde{H}_{Q-R}=\sum_{i=1}^{2} K_{i}\left(A \sigma_{+}^{(i)}+A^{\dagger} \sigma_{-}^{(i)}\right)$.

Thus, choosing $\omega_{1}=\omega_{2}=\tilde{\Omega}, \beta_{1}=\beta_{2} \equiv \beta$ and taking into account also the interaction of each subsystem (the two qubits and the nonlinear bus) with its own reservoir, it is possible to write the total Hamiltonian of the closed system as follows:

$$
\begin{aligned}
H= & \tilde{H}_{S}+H_{B}+H_{S-B} \\
= & \frac{\tilde{\Omega}}{2} \sum_{i=1}^{2} \sigma_{z}^{(i)}+\tilde{\Omega} A^{\dagger} A+K \sum_{i=1}^{2}\left(A \sigma_{+}^{(i)}+A^{\dagger} \sigma_{-}^{(i)}\right) \\
& +\sum_{i=1}^{2} \sum_{k} \omega_{k}^{(i)} b_{k}^{\dagger(i)} b_{k}^{(i)}+\sum_{j} \omega_{j} c_{j}^{\dagger} c_{j}+\sum_{i=1}^{2} \sum_{k} \epsilon_{k}^{(i)} \sigma_{x}^{(i)}\left(b_{k}^{(i)}+b_{k}^{\dagger(i)}\right) \\
& +\left(A+A^{\dagger}\right) \sum_{j}\left(c_{j}+c_{j}^{\dagger}\right) .
\end{aligned}
$$

\subsection{Eigenstates and eigenvalues of $H_{S}$}

In the representation of the operators $A$ and $A^{\dagger}$,

$$
\tilde{H}_{S}=\frac{\tilde{\Omega}}{2} \sum_{i=1}^{2} \sigma_{z}^{(i)}+\tilde{\Omega} A^{\dagger} A+K \sum_{i=1}^{2}\left(A \sigma_{+}^{(i)}+A^{\dagger} \sigma_{-}^{(i)}\right)
$$

has the well-known form of the Hamiltonian describing two two-level systems resonantly interacting with a linear bosonic mode. It is thus easy to find its eigenstates and eigenvalues in each invariant Hilbert subspace of the excitation number operator $\hat{n}=\frac{1}{2}\left(\sigma_{z}^{1}+\sigma_{z}^{2}\right)+A^{\dagger} A+1$. In particular, we find that the eigensolutions of the Hamiltonian $\tilde{H}_{S}$ of a tripartite qubit-mediator system, schematically illustrated in Fig. 1, can be written down as follows:

- Subspace with $n=0$ excitations

$$
E_{0}=-\tilde{\Omega} \quad\left|\psi_{0}\right\rangle=|0--\rangle ;
$$

- Subspace with $n=1$ excitations

$$
\begin{array}{cl}
E_{1}^{(-)}=-\sqrt{2} K & \left|\psi_{1}^{-}\right\rangle=\frac{\sqrt{2}}{2}|1--\rangle-\frac{1}{2}|0+-\rangle-\frac{1}{2}|0-+\rangle, \\
E_{1}^{(0)}=0 & \left|\psi_{1}^{0}\right\rangle=-\frac{1}{\sqrt{2}}|0+-\rangle+\frac{1}{\sqrt{2}}|0-+\rangle, \\
E_{1}^{(+)}=\sqrt{2} K & \left|\psi_{1}^{+}\right\rangle=\frac{\sqrt{2}}{2}|1--\rangle+\frac{1}{2}|0+-\rangle+\frac{1}{2}|0-+\rangle ;
\end{array}
$$

- Subspace with $n \geq 2$ excitations 
Fig. 1 Schematic illustration of eigenstates of tripartite qubit-mediator system; states are ordered for increasing energies from bottom to top; degenerate states appear at same height

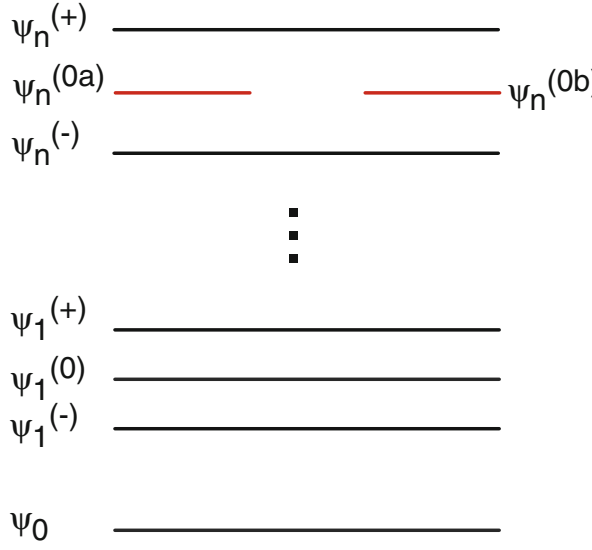

$$
\begin{aligned}
& E_{n}^{(-)}=\tilde{\Omega}(n-1)-K \sqrt{2(2 n-1)}, \\
& \left|\psi_{n}^{-}\right\rangle=\sqrt{\frac{n}{2(2 n-1)}}|n--\rangle \text {, } \\
& -\frac{1}{2}(|n-1,+-\rangle+|n-1,-+\rangle)+\sqrt{\frac{n-1}{2(2 n-1)}}|n-2,++\rangle, \\
& E_{n}^{(0)}=\tilde{\Omega}(n-1), \\
& \left|\psi_{n}^{0 a}\right\rangle=-\sqrt{\frac{n-1}{2 n-1}}|n--\rangle+\sqrt{\frac{n}{2 n-1}}|n-2,++\rangle, \\
& \left|\psi_{n}^{0 b}\right\rangle=-\frac{1}{\sqrt{2}}|n-1,+-\rangle+\frac{1}{\sqrt{2}}|n-1,-+\rangle, \\
& E_{n}^{(+)}=\tilde{\Omega}(n-1)+K \sqrt{2(2 n-1)}, \\
& \left|\psi_{n}^{+}\right\rangle=\sqrt{\frac{n}{2(2 n-1)}}|n--\rangle \text {, } \\
& +\frac{1}{2}(|n-1,+-\rangle+|n-1,-+\rangle)+\sqrt{\frac{n-1}{2(2 n-1)}}|n-2,++\rangle \text {. }
\end{aligned}
$$

\section{System master equation}

Once the eigensolutions of the free Hamiltonian of the system are known, the general approach [13, p. 133] to derive the master equation requires as a second step the construction of the dissipative jump operators describing transitions between the eigenstates of the system, taking into account possible degeneracies of the Bohr transition frequencies. More specifically, if the complete Hamiltonian of the open quantum system is written as

$H=H_{S}+H_{B}+H_{\text {int }}$,

where $H_{S}$ is the free Hamiltonian of the system, $H_{B}$ the Hamiltonian of the bath, and

$H_{\text {int }}=\sum_{\alpha} S_{\alpha} \otimes B_{\alpha}$,

where $S_{\alpha}$ acts on the system Hilbert space whereas $B_{\alpha}$ acts on the bath Hilbert space, then one can decompose $S_{\alpha}$ as follows: 
$S_{\alpha}=\sum_{\omega} S_{\alpha}(\omega)$,

with

$S_{\alpha}(\omega)=\sum_{E-E^{\prime}=\omega} \prod\left(E^{\prime}\right) S_{\alpha} \prod(E)$,

where $\prod(E)$ is the projection operator onto the eigenspace belonging to the eigenvalue $E$ and $\omega$ the set of all possible transition frequencies. In correspondence to each $S_{\alpha}(\omega)$ it is possible to construct a "Lindblad" term in the microscopic master equation given by [13, p. 136]

$\gamma_{\alpha}(\omega)\left(S_{\alpha}(\omega) \rho S_{\alpha}^{\dagger}(\omega)-\frac{1}{2}\left\{S_{\alpha}^{\dagger}(\omega) S_{\alpha}(\omega), \rho\right\}\right)$

having assumed that the reservoirs are uncorrelated and where the symbol $\{X, Y\}=X Y+Y X$ is the anticommutator between generic operators $X$ and $Y$. In Eq. (15) the decay rates $\gamma_{\alpha}(\omega)$ can be expressed in terms of appropriate environment correlation functions and thus depend on the statistical properties of its spectrum. At the moment, we make no assumptions concerning this point, reserving the possibility to define the spectral properties of the three baths when we solve the dynamics of the system starting from a given initial condition.

We now apply this formalism to our case constructing the proper Lindblad terms for each Bohr transition frequency. Let us concentrate, for example, on the details of the construction of the Lindblad term corresponding to the transition frequency $\omega=\tilde{\Omega}$, describing jumps between the subspace with $n$ excitations and that characterized by $n-1$ excitations. Taking into account the spectrum of $\tilde{H}_{S}$, as previously given, it is possible to convince oneself that, setting $S_{F}=A, S_{S 1}=\sigma_{x}^{(1)}$, and $S_{S 2}=\sigma_{x}^{(2)}$, we have

$$
\begin{aligned}
S_{F}(\tilde{\Omega})= & \left|\psi_{1}^{0}\right\rangle\left\langle\psi_{2}^{0 b}\right| \\
& +\sum_{n \geq 3} 2 \sqrt{\frac{n(n-1)(n-2)}{(2 n-1)(2 n-3)}}\left|\psi_{n-1}^{0 a}\right\rangle\left\langle\psi_{n}^{0 a}|+\sqrt{n-1}| \psi_{n-1}^{0 b}\right\rangle\left\langle\psi_{n}^{0 b}\right|,
\end{aligned}
$$

$$
\begin{aligned}
S_{S 1}(\tilde{\Omega})= & -\frac{1}{\sqrt{2}}\left|\psi_{0}\right\rangle\left\langle\psi_{1}^{0}\left|+\frac{1}{\sqrt{3}}\right| \psi_{1}^{0}\right\rangle\left\langle\psi_{2}^{0 a}\right| \\
& +\sum_{n \geq 3} 2 \sqrt{\frac{n-2}{2(2 n-3)}}\left|\psi_{n-1}^{0 a}\right\rangle\left\langle\psi_{n}^{0 b}\left|+\sqrt{\frac{n}{2(2 n-1)}}\right| \psi_{n-1}^{0 b}\right\rangle\left\langle\psi_{n}^{0 a}\right|,
\end{aligned}
$$

and, finally,

$$
\begin{aligned}
S_{S 2}(\tilde{\Omega})= & \frac{1}{\sqrt{2}}\left|\psi_{0}\right\rangle\left\langle\psi_{1}^{0}\left|-\frac{1}{\sqrt{3}}\right| \psi_{1}^{0}\right\rangle\left\langle\psi_{2}^{0 a}\right| \\
& +\sum_{n \geq 3} 2 \sqrt{\frac{n-2}{2(2 n-3)}}\left|\psi_{n-1}^{0 a}\right\rangle\left\langle\psi_{n}^{0 b}\left|+\sqrt{\frac{n}{2(2 n-1)}}\right| \psi_{n-1}^{0 b}\right\rangle\left\langle\psi_{n}^{0 a}\right| .
\end{aligned}
$$

Let us observe that in particular $S_{S 1}(\tilde{\Omega})=-S_{S 2}(\tilde{\Omega})$. Writing for $S_{i}(\tilde{\Omega})(i=F, S 1, S 2)$ the appropriate Lindblad term, as Eq. (15) requires, and repeating the same procedure for each Bohr transition frequency, we finally get the 
master equation governing the dissipative dynamics of our open quantum system. In particular, it is possible to demonstrate that the master equation of the system assumes the following form:

$$
\begin{aligned}
\dot{\rho}= & -\mathrm{i}\left[H_{S}, \rho\right] \\
& +\sum_{\ell= \pm} \Gamma_{1 \ell}\left(\left|\psi_{0}\right\rangle\left\langle\psi_{1}^{\ell}|\rho| \psi_{1}^{\ell}\right\rangle\left\langle\psi_{0}\left|-\frac{1}{2}\right| \psi_{1}^{\ell}\right\rangle\left\langle\psi_{1}^{\ell}\left|\rho-\frac{1}{2} \rho\right| \psi_{1}^{\ell}\right\rangle\left\langle\psi_{1}^{\ell}\right|\right) \\
& +\sum_{n \geq 2 ; \ell, \ell^{\prime}= \pm} \Gamma_{n, \ell \ell^{\prime}}\left(\left|\psi_{n-1}^{\ell^{\prime}}\right\rangle\left\langle\psi_{n}^{\ell}|\rho| \psi_{n}^{\ell}\right\rangle\left\langle\psi_{n-1}^{\ell^{\prime}}\left|-\frac{1}{2}\right| \psi_{n}^{\ell}\right\rangle\left\langle\psi_{n}^{\ell}\left|\rho-\frac{1}{2} \rho\right| \psi_{n}^{\ell}\right\rangle\left\langle\psi_{n}^{\ell}\right|\right) \\
& +\sum_{n \geq 2 ; \ell= \pm} \Gamma_{n, a \ell}\left(\left|\psi_{n-1}^{\ell}\right\rangle\left\langle\psi_{n}^{0 a}|\rho| \psi_{n}^{0 a}\right\rangle\left\langle\psi_{n-1}^{\ell}\left|-\frac{1}{2}\right| \psi_{n}^{0 a}\right\rangle\left\langle\psi_{n}^{0 a}\left|\rho-\frac{1}{2} \rho\right| \psi_{n}^{0 a}\right\rangle\left\langle\psi_{n}^{0 a}\right|\right) \\
& +\sum_{n \geq 2 ; \ell= \pm} \Gamma_{n, b \ell}\left(\left|\psi_{n-1}^{\ell}\right\rangle\left\langle\psi_{n}^{0 b}|\rho| \psi_{n}^{0 b}\right\rangle\left\langle\psi_{n-1}^{\ell}\left|-\frac{1}{2}\right| \psi_{n}^{0 b}\right\rangle\left\langle\psi_{n}^{0 b}\left|\rho-\frac{1}{2} \rho\right| \psi_{n}^{0 b}\right\rangle\left\langle\psi_{n}^{0 b}\right|\right) \\
& +\sum_{n \geq 2 ; \ell= \pm} \Gamma_{n, a b \ell}\left(\left|\psi_{n-1}^{\ell}\right\rangle\left\langle\psi_{n}^{0 b}|\rho| \psi_{n}^{0 a}\right\rangle\left\langle\psi_{n-1}^{\ell}|+| \psi_{n-1}^{\ell}\right\rangle\left\langle\psi_{n}^{0 a}|\rho| \psi_{n}^{0 b}\right\rangle\left\langle\psi_{n-1}^{\ell}\right|\right. \\
& \left.-\frac{1}{2}\left(\left|\psi_{n}^{0 a}\right\rangle\left\langle\psi_{n}^{0 b}|\rho+\rho| \psi_{n}^{0 a}\right\rangle\left\langle\psi_{n}^{0 b}|+| \psi_{n}^{0 b}\right\rangle\left\langle\psi_{n}^{0 a}|\rho+\rho| \psi_{n}^{0 b}\right\rangle\left\langle\psi_{n}^{0 a}\right|\right)\right) \\
& +\sum_{n \geq 2 ; \ell= \pm} \Gamma_{n, \ell a}\left(\left|\psi_{n-1}^{0 a}\right\rangle\left\langle\psi_{n}^{\ell}|\rho| \psi_{n}^{\ell}\right\rangle\left\langle\psi_{n-1}^{0 a}\left|-\frac{1}{2}\right| \psi_{n}^{\ell}\right\rangle\left\langle\psi_{n}^{\ell}\left|\rho-\frac{1}{2} \rho\right| \psi_{n}^{\ell}\right\rangle\left\langle\psi_{n}^{\ell}\right|\right) \\
& +\sum_{n \geq 2 ; \ell= \pm} \Gamma_{n, \ell b}\left(\left|\psi_{n-1}^{0 b}\right\rangle\left\langle\psi_{n}^{\ell}|\rho| \psi_{n}^{\ell}\right\rangle\left\langle\psi_{n-1}^{0 b}\left|-\frac{1}{2}\right| \psi_{n}^{\ell}\right\rangle\left\langle\psi_{n}^{\ell}\left|\rho-\frac{1}{2} \rho\right| \psi_{n}^{\ell}\right\rangle\left\langle\psi_{n}^{\ell \ell}\right|\right) \\
& +\sum_{n \geq 2 ; \ell= \pm} \Gamma_{n, \ell a b}\left(\left|\psi_{n-1}^{0 a}\right\rangle\left\langle\psi_{n}^{\ell}|\rho| \psi_{n}^{\ell}\right\rangle\left\langle\psi_{n-1}^{0 b}|+| \psi_{n-1}^{0 b}\right\rangle\left\langle\psi_{n}^{\ell}|\rho| \psi_{n}^{\ell}\right\rangle\left\langle\psi_{n-1}^{0 a}\right|\right) \\
& +\mathcal{D}_{F}(\tilde{\Omega})+\mathcal{D}_{S}(\tilde{\Omega}),
\end{aligned}
$$

where

$$
\begin{aligned}
& \mathcal{D}_{F}(\tilde{\Omega})=\gamma_{F}(\tilde{\Omega})\left\{\left|\psi_{1}^{0}\right\rangle\left\langle\psi_{2}^{0 b}|\rho| \psi_{2}^{0 b}\right\rangle\left\langle\psi_{1}^{0}\right|\right. \\
& +\sum_{n \geq 3}\left[2 \sqrt{\frac{n(n-1)(n-2)}{(2 n-1)(2 n-3)}}\left(\left|\psi_{1}^{0}\right\rangle\left\langle\psi_{2}^{0 b}|\rho| \psi_{n}^{0 a}\right\rangle\left\langle\psi_{n-1}^{0 a}|+| \psi_{n-1}^{0 a}\right\rangle\left\langle\psi_{n}^{0 a}|\rho| \psi_{2}^{0 b}\right\rangle\left\langle\psi_{1}^{0}\right|\right)\right. \\
& \left.+\sqrt{n-1}\left(\left|\psi_{1}^{0}\right\rangle\left\langle\psi_{2}^{0 b}|\rho| \psi_{n}^{0 b}\right\rangle\left\langle\psi_{n-1}^{0 b}|+| \psi_{n-1}^{0 b}\right\rangle\left\langle\psi_{n}^{0 b}|\rho| \psi_{2}^{0 b}\right\rangle\left\langle\psi_{1}^{0}\right|\right)\right] \\
& +\sum_{n, n^{\prime} \geq 3}\left[\sqrt[4]{\frac{n(n-1)(n-2)}{(2 n-1)(2 n-3)}} \sqrt{\frac{n^{\prime}\left(n^{\prime}-1\right)\left(n^{\prime}-2\right)}{\left(2 n^{\prime}-1\right)\left(2 n^{\prime}-3\right)}}\left|\psi_{n-1}^{0 a}\right\rangle\left\langle\psi_{n}^{0 a}|\rho| \psi_{n}^{\prime 0 a}\right\rangle\left\langle\psi_{n^{\prime}-1}^{0 a}\right|\right. \\
& +2 \sqrt{n^{\prime}-1} \sqrt{\frac{n(n-1)(n-2)}{(2 n-1)(2 n-3)}}\left|\psi_{n-1}^{0 a}\right\rangle\left\langle\psi_{n}^{0 a}|\rho| \psi_{n}^{\prime 0 b}\right\rangle\left\langle\psi_{n^{\prime}-1}^{0 b}\right| \\
& +2 \sqrt{n-1} \sqrt{\frac{n^{\prime}\left(n^{\prime}-1\right)\left(n^{\prime}-2\right)}{\left(2 n^{\prime}-1\right)\left(2 n^{\prime}-3\right)}}\left|\psi_{n-1}^{0 b}\right\rangle\left\langle\psi_{n}^{0 b}|\rho| \psi_{n}^{\prime 0 a}\right\rangle\left\langle\psi_{n^{\prime}-1}^{0 a}\right| \\
& \left.+\sqrt{(n-1)\left(n^{\prime}-1\right)}\left|\psi_{n-1}^{0 b}\right\rangle\left\langle\psi_{n}^{0 b}|\rho| \psi_{n}^{\prime 0 b}\right\rangle\left\langle\psi_{n^{\prime}-1}^{0 b}\right|\right] \\
& -\frac{1}{2}\left[\left|\psi_{2}^{0 b}\right\rangle\left\langle\psi_{2}^{0 b}|\rho+\rho| \psi_{2}^{0 b}\right\rangle\left\langle\psi_{2}^{0 b}\right|\right.
\end{aligned}
$$




$$
\left.\left.+\sum_{n \geq 3}\left(4 \frac{n(n-1)(n-2)}{(2 n-1)(2 n-3)}\left|\psi_{n}^{0 a}\right\rangle\left\langle\psi_{n}^{0 a}|\rho+\rho| \psi_{n}^{0 a}\right\rangle\left\langle\psi_{n}^{0 a}|+(n-1)| \psi_{n}^{0 b}\right\rangle\left\langle\psi_{n}^{0 b}|\rho+\rho| \psi_{n}^{0 b}\right\rangle\left\langle\psi_{n}^{0 b}\right|\right)\right]\right\}
$$

and

$$
\begin{aligned}
& \mathcal{D}_{S}(\tilde{\Omega})=\left[\gamma_{S 1}(\tilde{\Omega})+\gamma_{S 2}(\tilde{\Omega})\right]\left\{\frac{1}{2}\left|\psi_{0}\right\rangle\left\langle\psi_{1}^{0}|\rho| \psi_{1}^{0}\right\rangle\left\langle\psi_{0}\right|\right. \\
& -\frac{1}{\sqrt{6}}\left|\psi_{0}\right\rangle\left\langle\psi_{1}^{0}|\rho| \psi_{2}^{0 a}\right\rangle\left\langle\psi_{1}^{0}\left|-\frac{1}{\sqrt{6}}\right| \psi_{1}^{0}\right\rangle\left\langle\psi_{2}^{0 a}|\rho| \psi_{1}^{0}\right\rangle\left\langle\psi_{0}\right| \\
& +\frac{1}{3}\left|\psi_{1}^{0}\right\rangle\left\langle\psi_{2}^{0 a}|\rho| \psi_{2}^{0 a}\right\rangle\left\langle\psi_{1}^{0}\right|+\sum_{n \geq 3}\left(-\frac{1}{2} \sqrt{\frac{n-2}{2 n-3}}\left|\psi_{n-1}^{0 a}\right\rangle\left\langle\psi_{n}^{0 b}|\rho| \psi_{1}^{0}\right\rangle\left\langle\psi_{0}\right|\right. \\
& -\frac{1}{2} \sqrt{\frac{n}{2 n-1}}\left|\psi_{n-1}^{0 b}\right\rangle\left\langle\psi_{n}^{0 a}|\rho| \psi_{1}^{0}\right\rangle\left\langle\psi_{0}\right| \\
& +\sqrt{\frac{n-2}{6(2 n-3)}}\left|\psi_{n-1}^{0 a}\right\rangle\left\langle\psi_{n}^{0 b}|\rho| \psi_{2}^{0 a}\right\rangle\left\langle\psi_{1}^{0}\left|+\sqrt{\frac{n}{6(2 n-1)}}\right| \psi_{n-1}^{0 b}\right\rangle\left\langle\psi_{n}^{0 a}|\rho| \psi_{2}^{0 a}\right\rangle\left\langle\psi_{1}^{0}\right| \\
& -\frac{1}{2} \sqrt{\frac{n-2}{2 n-3}}\left|\psi_{0}\right\rangle\left\langle\psi_{1}^{0}|\rho| \psi_{n}^{0 b}\right\rangle\left\langle\psi_{n-1}^{0 a}\left|-\frac{1}{2} \sqrt{\frac{n}{2 n-1}}\right| \psi_{0}\right\rangle\left\langle\psi_{1}^{0}|\rho| \psi_{n}^{0 a}\right\rangle\left\langle\psi_{n-1}^{0 b}\right| \\
& \left.+\sqrt{\frac{n-2}{6(2 n-3)}}\left|\psi_{1}^{0}\right\rangle\left\langle\psi_{2}^{0 a}|\rho| \psi_{n}^{0 b}\right\rangle\left\langle\psi_{n-1}^{0 a}\left|+\sqrt{\frac{n}{6(2 n-1)}}\right| \psi_{1}^{0}\right\rangle\left\langle\psi_{2}^{0 a}|\rho| \psi_{n}^{0 a}\right\rangle\left\langle\psi_{n-1}^{0 b}\right|\right) \\
& +\sum_{n, n^{\prime} \geq 3}\left(\sqrt{\frac{n-2}{2(2 n-3)}} \sqrt{\frac{n^{\prime}-2}{2\left(2 n^{\prime}-3\right)}}\left|\psi_{n-1}^{0 a}\right\rangle\left\langle\psi_{n}^{0 b}|\rho| \psi_{n^{\prime}}^{0 b}\right\rangle\left\langle\psi_{n^{\prime}-1}^{0 a}\right|\right. \\
& +\sqrt{\frac{n-2}{2(2 n-3)}} \sqrt{\frac{n^{\prime}}{2\left(2 n^{\prime}-1\right)}}\left|\psi_{n-1}^{0 a}\right\rangle\left\langle\psi_{n}^{0 b}|\rho| \psi_{n^{\prime}}^{0 a}\right\rangle\left\langle\psi_{n^{\prime}-1}^{0 b}\right| \\
& +\sqrt{\frac{n}{2(2 n-1)}} \sqrt{\frac{n^{\prime}-2}{2\left(2 n^{\prime}-3\right)}}\left|\psi_{n-1}^{0 b}\right\rangle\left\langle\psi_{n}^{0 a}|\rho| \psi_{n^{\prime}}^{0 b}\right\rangle\left\langle\psi_{n^{\prime}-1}^{0 a}\right| \\
& \left.+\sqrt{\frac{n}{2(2 n-1)}} \sqrt{\frac{n^{\prime}}{2\left(2 n^{\prime}-1\right)}}\left|\psi_{n-1}^{0 b}\right\rangle\left\langle\psi_{n}^{0 a}|\rho| \psi_{n^{\prime}}^{0 a}\right\rangle\left\langle\psi_{n^{\prime}-1}^{0 b}\right|\right) \\
& -\frac{1}{2}\left[\frac{1}{2}\left|\psi_{1}^{0}\right\rangle\left\langle\psi_{1}^{0}\left|\rho+\frac{1}{2} \rho\right| \psi_{1}^{0}\right\rangle\left\langle\psi_{1}^{0}\left|+\frac{1}{3}\right| \psi_{2}^{0 a}\right\rangle\left\langle\psi_{2}^{0 a}\left|\rho+\frac{1}{3} \rho\right| \psi_{2}^{0 a}\right\rangle\left\langle\psi_{2}^{0 a}\right|\right. \\
& +\sum n \geq 3\left(\frac{n-2}{2(2 n-3)}\left(\left|\psi_{n}^{0 b}\right\rangle\left\langle\psi_{n}^{0 b}|\rho+\rho| \psi_{n}^{0 b}\right\rangle\left\langle\psi_{n}^{0 b}\right|\right)\right. \\
& \left.\left.\left.+\frac{n}{2(2 n-1)}\left(\left|\psi_{n}^{0 a}\right\rangle\left\langle\psi_{n}^{0 a}|\rho+\rho| \psi_{n}^{0 a}\right\rangle\left\langle\psi_{n}^{0 a}\right|\right)\right)\right]\right\} \text {. }
\end{aligned}
$$

The coefficients $\Gamma$ appearing in Eq. (19) are appropriate combinations of the decay rates $\gamma_{i}(\omega)$ and, for our convenience, are explicitly given in the appendix.

\section{System dynamics}

Exploiting the knowledge of the system master equation, as derived in the previous section, we can now analyze its dynamics starting, for example, from the initial condition $\left|\psi_{0}\right\rangle=|0+-\rangle$, where the nonlinear mode is in its vacuum state $(|0\rangle)$, the qubit 1 is in its upper level $(|+\rangle)$, and the other qubit is in its ground state $(|-\rangle)$. Taking into 

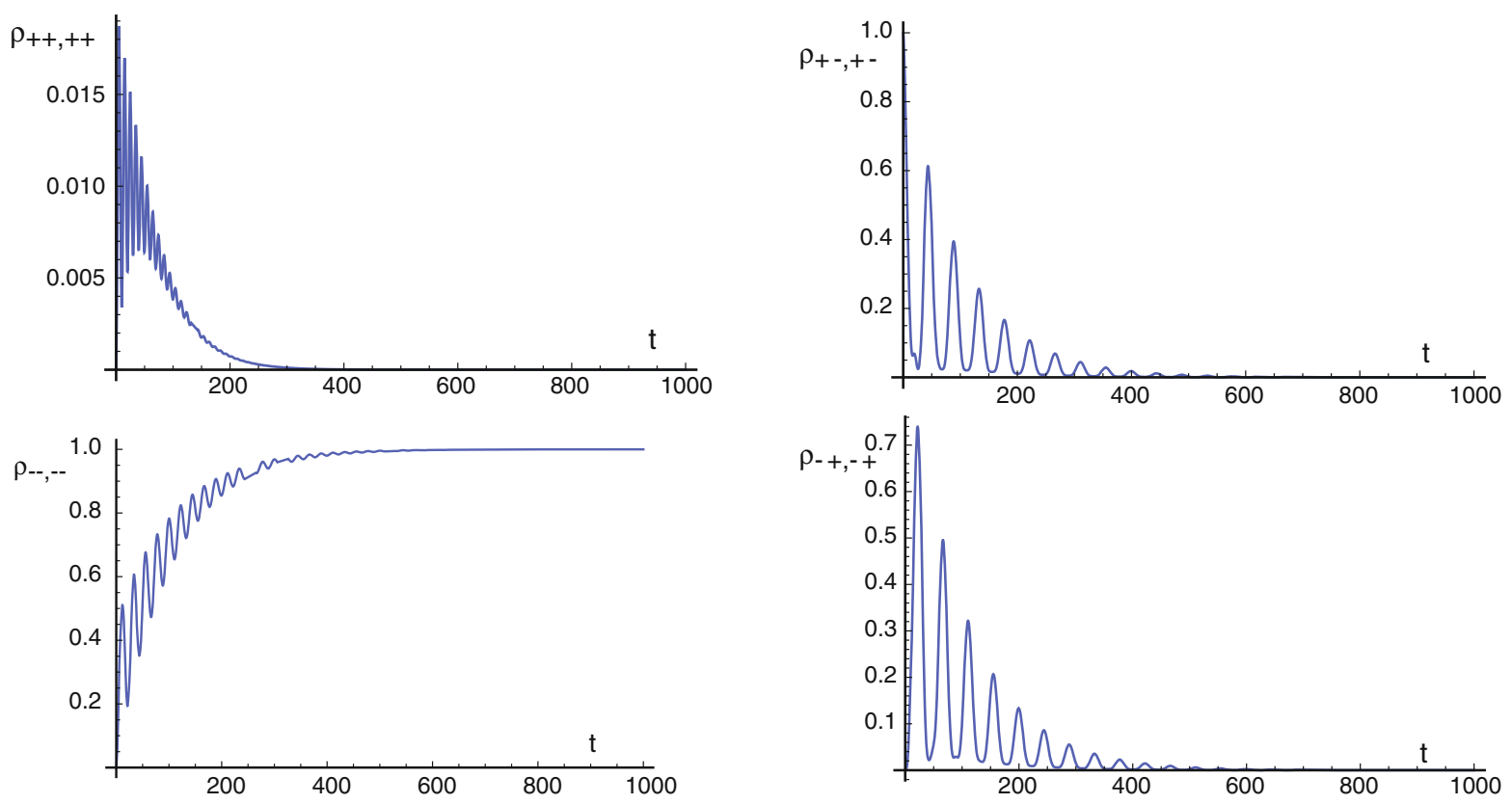

Fig. 2 Time evolution (time in units of $\Omega^{-1}$ ) of the population of the states $|++\rangle$ (up) and $|--\rangle$ (down), calculated choosing $\gamma=0.01 \Omega$ and $K=0.1 \Omega$. The state is represented by the density operator $\rho$, obtained by a numerical solution of Eq. (19)

Fig. 3 Time evolution (time in units of $\Omega^{-1}$ ) of the population of the states $|+-\rangle$ (up) and $|-+\rangle$ (down), calculated choosing $\gamma=0.01 \Omega$ and $K=0.1 \Omega$. The state is represented by the density operator $\rho$, obtained by a numerical solution of Eq. (19)

account that the master equation was constructed starting from $\tilde{H}_{S}$ instead of $H_{S}$, we must first express the initial state of the mediator in terms of the eigenstates of the operator $A$ defined in Eq. (2). To this end, we can exploit the unitary operator

$U=\mathrm{e}^{\frac{z}{2}\left(a^{2}-a^{\dagger 2}\right)}$,

with $z=\frac{1}{2} \ln (\tilde{\Omega} / \Omega)(z \in \mathbb{R})$, observing that

$A=U a U^{\dagger}$.

In view of Eq. (23), indeed, indicating by $|\tilde{n}\rangle$ the eigenstates of the transformed number operator $A^{\dagger} A$, we can write $|n\rangle=U^{\dagger}|\tilde{n}\rangle$. Thus we can express the initial state of the mediator as follows:

$|0\rangle=\sum_{m}^{\infty} P_{m}^{1 / 2}|\widetilde{2 m}\rangle$,

with

$P_{m}=\left(1-z^{2}\right)^{1 / 2} \frac{2 m !}{2^{2 m}(m !)^{2}} z^{2 m}$,

where we have used the action of the squeezing operator in Eq. (22) on the vacuum state [15]. In what follows we suppose that the parameter $\alpha$ describing the strength of the nonlinearity of the bosonic mode is fixed to the value $\alpha=0.1 \omega$. In such a condition it is possible to prove that $|0\rangle \approx P_{0}^{1 / 2}|\widetilde{0}\rangle+P_{1}^{1 / 2}|\widetilde{2}\rangle$. In other words, the initial state of the tripartite system is $\left|\psi_{0}\right\rangle \approx w_{0}|\tilde{0}+-\rangle+w_{1}|\tilde{2}+-\rangle$, with $w_{0}=\sqrt{\frac{P_{0}}{P_{0}+P_{1}}}$ and $w_{1}=\sqrt{1-w_{0}^{2}}$ or, expressed in terms of eigenstates of $\tilde{H}_{S}$, 
Fig. 4 Time evolution (time in units of $\Omega^{-1}$ ) of the function concurrence in Eq. (27), calculated choosing $\gamma=0.01 \Omega$ and $K=0.1 \Omega$.

The state is represented by the density operator $\rho$, obtained by a numerical solution of Eq. (19)

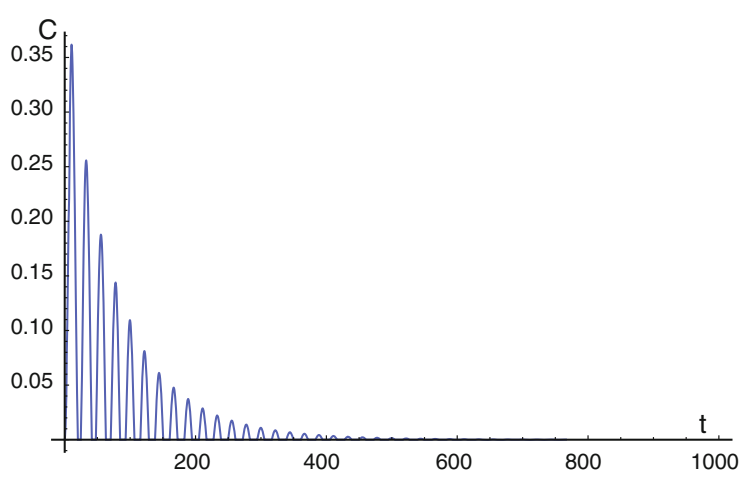

$$
\begin{aligned}
\left|\psi_{0}\right\rangle \approx & \frac{w_{0}}{2}\left[-\left|\psi_{1}^{-1}\right\rangle+\left|\psi_{1}^{1}\right\rangle-\sqrt{2}\left|\psi_{1}^{0}\right\rangle\right] \\
& +\frac{w_{1}}{2}\left[\left|\psi_{3}^{1}\right\rangle-\sqrt{2}\left|\psi_{3}^{0 b}\right\rangle-\left|\psi_{3}^{-1}\right\rangle\right] .
\end{aligned}
$$

After very long calculations, it is thus possible to derive the time evolution of the initial state, starting from Eq. (19). In particular, we have numerically solved the dynamics of the system supposing that the three baths are at zero temperature and are characterized by the same flat spectrum that in turn implies setting $\gamma_{F}(\omega)=\gamma_{S 1}(\omega)=$ $\gamma_{S 2}(\omega)=\gamma$.

Here we confine ourselves to the analysis of the bipartite qubit 1 -qubit 2 subsystem by looking at the time evolution of the populations and of the entanglement established between them via their common interaction with the mediator. As a measure of the entanglement we choose the well-known concurrence function $\mathcal{C}$, which is well suited for a system of two qubits; in our case this coincides with

$$
\mathcal{C}=2 \max \left\{0,\left|\rho_{++,--}\right|-\sqrt{\rho_{+-,+-} \rho_{-+,-+}},\left|\rho_{+-,-+}\right|-\sqrt{\rho_{++,++} \rho_{--,--}}\right\},
$$

where $\rho_{i j, k l}$ is the matrix element $\langle i j|\rho| k l\rangle(i, j, k, l \equiv+,-)$ of the reduced density matrix describing the dynamics of the qubit-qubit system.

As expected, Figs. 2 and 3 illustrate the excitation exchanges between the two qubits mediated by the presence of the nonlinear mode. The bipartite system, initially prepared in the state $|+-\rangle$, decays toward its own ground state $|--\rangle$ due to the presence of the external environment. The oscillations between the populations of states $|+-\rangle$ and $|-+\rangle$ are mirrored in the oscillations of the entanglement established between the two qubits. Such oscillations can be quantified by the concurrence of the two qubits, as shown in Fig. 4.

\section{Conclusions}

In this paper we focused our attention on the construction of a microscopic master equation describing the dissipative dynamics of system of two qubits in interaction via their common coupling with a nonlinear mediator. We showed that the construction of the microscopic master equation is more involved than simply adding ad hoc terms in the dissipative part of the evolution equation. Exploiting this master equation, we analyzed the system dynamics demonstrating that, for decoherence times in agreement with recent experimental findings, the coherent coupling established between the two qubits survives in the presence of dissipation. However, the behavior of the concurrence is what one would intuitively expect. For this reason, the question of whether it is really worthwhile to use the microscopic model instead of the phenomenological one for the specific system under consideration remains an open problem. The search for initial conditions leading to discrepancies between the microscopic and the phenomenological models will be the object of our future research.

Acknowledgments The authors thank Prof. E. Zipper for useful discussions. Financial support from the Italian Project PRIN 2008C3JE43_003 is acknowledged. 


\section{Appendix}

In this section we give the explicit dependence of the coefficient $\Gamma$ appearing in the system master equation as a function of the decay rates $\gamma_{i}(i=F, S 1, S 2)$ :

$$
\begin{aligned}
& \Gamma_{1 \ell}=\frac{1}{2} \gamma_{F}\left(E_{1}^{\ell}-E_{0}\right) \\
& +\frac{1}{4}\left[\gamma_{S 1}\left(E_{1}^{\ell}-E_{0}\right)+\gamma_{S 2}\left(E_{+}^{\ell}-E_{0}\right)\right] \quad \ell= \pm 1, \\
& \Gamma_{n++}=\gamma_{F}\left(E_{n}^{+}-E_{n-1}^{+}\right)\left[(n-1) \sqrt{\frac{n-1}{(2 n-1)(2 n-3)}}+\frac{\sqrt{n-1}}{2}\right]^{2} \\
& +\frac{1}{4}\left(\gamma_{S 1}\left(E_{n}^{+}-E_{n-1}^{+}\right)+\gamma_{S 2}\left(E_{n}^{+}-E_{n-1}^{+}\right)\right)\left[\sqrt{\frac{n-1}{2(2 n-3)}}+\sqrt{\frac{n-1}{2(2 n-1)}}\right]^{2}, \\
& \Gamma_{n+-}=\gamma_{F}\left(E_{n}^{+}-E_{n-1}^{-}\right)\left[(n-1) \sqrt{\frac{n-1}{(2 n-1)(2 n-3)}}-\frac{\sqrt{n-1}}{2}\right]^{2} \\
& +\frac{1}{4}\left(\gamma_{S 1}\left(E_{n}^{+}-E_{n-1}^{-}\right)+\gamma_{S 2}\left(E_{n}^{+}-E_{n-1}^{-}\right)\right)\left[\sqrt{\frac{n-1}{2(2 n-3)}}-\sqrt{\frac{n-1}{2(2 n-1)}}\right]^{2}, \\
& \Gamma_{n-+}=\gamma_{F}\left(E_{n}^{-}-E_{n-1}^{+}\right)\left[(n-1) \sqrt{\frac{n-1}{(2 n-1)(2 n-3)}}-\frac{\sqrt{n-1}}{2}\right]^{2} \\
& +\frac{1}{4}\left(\gamma_{S 1}\left(E_{n}^{-}-E_{n-1}^{+}\right)+\gamma_{S 2}\left(E_{n}^{-}-E_{n-1}^{+}\right)\right)\left[\sqrt{\frac{n-1}{2(2 n-3)}}-\sqrt{\frac{n-1}{2(2 n-1)}}\right]^{2} \\
& \Gamma_{n--}=\gamma_{F}\left(E_{n}^{-}-E_{n-1}^{-}\right)\left[(n-1) \sqrt{\frac{n-1}{(2 n-1)(2 n-3)}}+\frac{\sqrt{n-1}}{2}\right]^{2} \\
& +\frac{1}{4}\left(\gamma_{S 1}\left(E_{n}^{-}-E_{n-1}^{-}\right)+\gamma_{S 2}\left(E_{n}^{-}-E_{n-1}^{-}\right)\right)\left[\sqrt{\frac{n-1}{2(2 n-3)}}+\sqrt{\frac{n-1}{2(2 n-1)}}\right]^{2}, \\
& \Gamma_{n a+}=\gamma_{F}\left(E_{n}^{0}-E_{n-1}^{+}\right) \frac{n}{2(2 n-1)(2 n-3)} \\
& +\frac{1}{4}\left(\gamma_{S 1}\left(E_{n}^{0}-E_{n-1}^{+}\right)+\gamma_{S 2}\left(E_{n}^{0}-E_{n-1}^{+}\right)\right) \frac{n}{2 n-1}, \\
& \Gamma_{n b+}=\frac{1}{4}\left(\gamma_{S 1}\left(E_{n}^{0}-E_{n-1}^{+}\right)+\gamma_{S 2}\left(E_{n}^{0}-E_{n-1}^{+}\right)\right) \frac{n-1}{2 n-3} \\
& \Gamma_{n a b+}=-\frac{1}{4}\left(\gamma_{S 1}\left(E_{n}^{0}-E_{n-1}^{+}\right)-\gamma_{S 2}\left(E_{n}^{0}-E_{n-1}^{+}\right)\right) \sqrt{\frac{n(n-1)}{(2 n-1)(2 n-3)}}, \\
& \Gamma_{n a-}=\gamma_{F}\left(E_{n}^{0}-E_{n-1}^{-}\right) \frac{n}{2(2 n-1)(2 n-3)}(n-2)^{2} \\
& +\frac{1}{4}\left(\gamma_{S 1}\left(E_{n}^{0}-E_{n-1}^{-}\right)+\gamma_{S 2}\left(E_{n}^{0}-E_{n-1}^{-}\right)\right) \frac{n}{2 n-1}, \\
& \Gamma_{n b-}=\frac{1}{4}\left(\gamma_{S 1}\left(E_{n}^{0}-E_{n-1}^{-}\right)+\gamma_{S 2}\left(E_{n}^{0}-E_{n-1}^{-}\right)\right) \frac{n-1}{2 n-3},
\end{aligned}
$$




$$
\begin{aligned}
& \Gamma_{n a b-}=\frac{1}{4}\left(\gamma_{S 1}\left(E_{n}^{0}-E_{n-1}^{-}\right)-\gamma_{S 2}\left(E_{n}^{0}-E_{n-1}^{-}\right)\right) \sqrt{\frac{n(n-1)}{(2 n-1)(2 n-3)}}, \\
& \Gamma_{n+a}=\gamma_{F}\left(E_{n}^{+}-E_{n-1}^{0}\right) \frac{n-2}{2(2 n-1)(2 n-3)} \\
& +\frac{1}{4}\left(\gamma_{S 1}\left(E_{n}^{+}-E_{n-1}^{0}\right)+\gamma_{S 2}\left(E_{n}^{+}-E_{n-1}^{0}\right)\right) \frac{n-2}{2 n-3}, \\
& \Gamma_{n+b}=\frac{1}{4}\left(\gamma_{S 1}\left(E_{n}^{+}-E_{n-1}^{0}\right)+\gamma_{S 2}\left(E_{n}^{+}-E_{n-1}^{0}\right)\right) \frac{n-1}{2 n-1}, \\
& \Gamma_{n+a b}=-\frac{1}{4}\left(\gamma_{S 1}\left(E_{n}^{+}-E_{n-1}^{0}\right)+\gamma_{S 2}\left(E_{n}^{+}-E_{n-1}^{0}\right)\right) \sqrt{\frac{(n-1)(n-2)}{(2 n-1)(2 n-3)}}, \\
& \Gamma_{n-a}=\gamma_{F}\left(E_{n}^{-}-E_{n-1}^{0}\right) \frac{n-2}{2(2 n-1)(2 n-3)} \\
& +\frac{1}{4}\left(\gamma_{S 1}\left(E_{n}^{-}-E_{n-1}^{0}\right)+\gamma_{S 2}\left(E_{n}^{-}-E_{n-1}^{0}\right)\right) \frac{n-2}{2 n-3}, \\
& \Gamma_{n-b}=\frac{1}{4}\left(\gamma_{S 1}\left(E_{n}^{-}-E_{n-1}^{0}\right)+\gamma_{S 2}\left(E_{n}^{-}-E_{n-1}^{0}\right)\right) \frac{n-1}{2 n-1}, \\
& \Gamma_{n-a b}=-\frac{1}{4}\left(\gamma_{S 1}\left(E_{n}^{-}-E_{n-1}^{0}\right)-\gamma_{S 2}\left(E_{n}^{-}-E_{n-1}^{0}\right)\right) \sqrt{\frac{(n-1)(n-2)}{(2 n-1)(2 n-3)}} \text {. }
\end{aligned}
$$

\section{References}

1. Migliore R, Yuasa K, Nakazato H, Messina A (2006) Generation of multipartite entangled states in Josephson architectures. Phys Rev B 74:104503

2. DiCarlo L, Reed MD, Sun L, Johnson BR, Chow JM, Gambetta JM, Frunzio L, Girvin SM, Devoret MH, Schoelkopf R (2010) Preparation and measurement of three-qubit entanglement in a superconducting circuit. Nature 467:574

3. Migliore R, Scala M, Napoli A, Yuasa K, Nakazato H, Messina A (2011) Dissipative effects on a generation scheme of a W state in an array of coupled Josephson junctions. J Phys B At Mol Opt Phys 44:075503

4. Devoret MH, Wallraff A, Martinis JM (2004) Superconducting qubits: a short, review. arXiv:cond-mat/0411174

5. Levitov LS, Orlando TP, Majer JB, Mooij JE (2001) Quantum spin chains and Majorana states in arrays of coupled qubits. arXiv:cond-mat/0108266

6. Spilla S, Migliore R, Scala M, Napoli A (2012) GHZ state generation of three Josephson qubits in the presence of bosonic baths. J Phys B At Mol Opt Phys 45:065501

7. Migliore R, Yuasa K, Guccione M, Nakazato H, Messina A (2007) Diffusion and transfer of entanglement in an array of inductively coupled flux qubits. Phys Rev B 76:052501

8. Altomare F, Park JI, Cicak K, Sillanpää MA, Allman MS, Li D, Sirois A, Strong JA, Whittaker JD, Simonds RW (2010) Tripartite interactions between two phase qubits and a resonant cavity. arXiv:1004.0026

9. Neeley M Biakzak RC, Lenander M, Lucero E, Mariantoni M, O’connell AR, Sank D, Wang H, Wides M, Wenner J, Yin Y, Yamamoto T, Cleland AN, Martinis JM (2010) Generation of three-qubit entangled states using superconducting phase qubits. arXiv: $1004.4246 \mathrm{v} 2$

10. Kurpas M, Dajka J, Zipper E (2009) Entanglement of qubits via a nonlinear resonator. J Phys Condens Matter 21:235602

11. Scala M, Migliore R, Messina A (2008) Dissipation and entanglement dynamics for two interacting qubits coupled to independent reservoirs. J Phys A Math Theor 41:435304

12. Scala M, Migliore R, Messina A, Sanchez-Soto LL (2011) Robust stationary entanglement of two coupled qubits in independent environments. Eur Phys J D 61:199

13. Breuer HP, Petruccione F (2002) The theory of open quantum systems. Oxford University Press, Oxford

14. Cohen-Tannoudji C, Diu B, Laloë F (1977) Quantum mechanics, vol 1. Wiley, New York

15. Obada ASF, Darwish M (2005) Non-linear squeezing of the vacuum and the one-photon states as realizations of the SU(1,1) group. J Opt B 7:57-65 